\title{
Chronisches Schmerzsyndrom
}

\section{Die somatische Betrachtung reicht nicht}

Chronische Schmerzen sollte man weder ausschließlich somatisch noch ausschließlich psychologisch angehen. In einem multimodalen Behandlungskonzept haben auch Opioide einen gewissen Stellenwert, aber nicht bei allen Patienten und nicht auf Dauer.

Der Einsatz von Opioiden in der Schmerztherapie hat in den letzten Jahren deutlich zugenommen, wobei der Anstieg vorwiegend der Verordnung bei Patienten mit Nichttumorschmerzen geschuldet ist, deren Zahl ständig steige, so Dr. Andreas Kopf, Berlin. Betroffen sei in Deutschland inzwischen jeder siebte Bundesbürger und jeder zwanzigste leide an chronisch invalidisierenden Schmerzen, wobei muskulo-skelettale Schmerzen im Vordergrund stehen.

\section{"Yellow Flags"erkennen}

Bei der Chronifizierung von Nichttumorschmerzen spielen biopsychosoziale Faktoren die entscheidende Rolle. Man könnte sagen: Nicht der Schmerz bestimmt das Leben dieser Patienten, sondern das Leben macht ihren Schmerz unerträglich. Kognition, Emotion und Verhalten beeinflussen das Schmerzerleben. Mit anderen Worten: Die affektive und sensorische Perzeption ist verändert. Deshalb ist es wich-

tig, Risikofaktoren für eine Chronifizierung, die sogenannten „Yellow Flags“ zu erkennen: Angst vor körperlicher Belastung, extreme Angst vor der Schmerzbedingten Beeinträchtigung, depressive Verstimmung, Vermeidungsverhalten, Schlafstörungen, Medikamenten missbrauch, überprotektiver Partner, hohes Inanspruchnahmeverhalten, Angst vor einem Malignom, mehrere sich widersprechende Diagnosen, schlechte ärztliche Kommunikation und Unzufriedenheit über vorausgegangene Behandlungen.

\section{Schwache Wirkung der Opioide}

Die analgetische Wirkung von Opioiden wird über spezifische Rezeptoren, insbesondere die $\mu$-Rezeptoren vermittelt. Diese Rezeptoren sind supraspinal, spinal und im peripheren Gewebe lokalisiert. „In der Langzeitanwendung sind Opioide bei Nichttumorschmerzen im Hinblick auf die analgetische Wirksamkeit nicht-steroidalen Analgetika und Paracetamol nicht überlegen", so Kopf. Mit allen diesen Substanzen könne eine Abnahme des Schmerzes um 10 Skalaeinheiten auf der 100-stufigen Schmerzskala erreicht werden, bei Einbeziehung der Placebo- und Kontexterfahrungen seien es 25 Einheiten.

Eine Behandlung mit Opioiden könne bei Arthrosen, diabetischer Polyneuropathie, Postzosterneuralgie und chronischen Rückenschmerzen erwogen werden. Bei primären Kopfschmerzen, die in Attacken auftreten, bei funktionellen oder somatoformen Störungen sowie bei Schmerzen, die der Patient besonders ausgeprägt in Abhängigkeit von situativen und psychischen Faktoren empfindet, sind Opioide nicht indiziert. Bei anderen Krankheitsbildern sollte eine Therapie mit opioidhaltigen Analgetika als individueller Therapieversuch gewertet werden.

„Ein wesentlicher Faktor für den Erfolg einer Opioidbehandlung scheint die Integration in ein multimodales Therapiekonzept zu sein", so Kopf.

\section{Risiken beachten}

Um die Risiken einer Opioidtherapie zu minimieren, müssen Wirksamkeit und Nebenwirkungen regelmäßig überprüft werden. Potenziell lebensbedrohliche, unerwünschte Arzneimittelwirkungen sind im Unterschied zu NSAR für Opioide auch bei Langzeitanwendung nicht bekannt. Neben Übelkeit und Obstipation kann es aber durch die sedierende Wirkung zu Gleichgewichtsstörungen und Stürzen kommen. Zudem besteht ein Risiko für kognitive Dysfunktionen oder depressive Verstimmungen. Zusätzlich kann die Fahrsicherheit beeinträchtigt sein. Auch können immunologische und endokrinologische Nebenwirkungen auftreten, wie das opioidinduzierte Testosterondefizit-Syndrom i.S. eines Hypogonadismus.

„Die zunehmende Verordnung von Opioiden ist auch mit einer zunehmenden Inzidenz von Missbrauch und $\mathrm{Ab}$ hängigkeit verbunden“, warnte Kopf. Andererseits führe selbst eine nicht-indikationsgerechte Langzeittherapie mit einem Opioid nicht immer zu einer Suchterkrankung.

\section{Fazit für die Praxis:}

- Bei der Chronifizierung von Schmerzen spielen biopsychosoziale Faktoren eine wichtige Rolle

- Daraus ergibt sich die Notwendigkeit für ein multimodales Therapiekonzept

- Die analgetische Wirkung der Opioide ist vergleichbar mit der nicht-opioidhaltiger Analgetika

- Sinnvoll sind Opioide bei Arthrose, diabetischer Polyneuropathie, Postzosterneuralgie und chronischen Rückenschmerzen

- Ansonsten sollten sie als individueller Therapieversuch bewertet werden

- Wirksamkeit und Nebenwirkungen müssen regelmäßig überprüft werden.

(Peter Stiefelhagen)

Praxis update, 29.4.2016 in Köln 